\title{
IMPACTOS DO USO DAS REDES SOCIAIS VIRTUAIS NA SAÚDE MENTAL DOS ADOLESCENTES: UMA REVISÃO SISTEMÁTICA DA LITERATURA
}

\section{Impacts of the use of virtual social networks on adolescents' mental health: A systematic review of literature}

Karlla Souza - Instituto Federal de Educação Ciência e Tecnologia de Alagoas /Brasil Mônica Ximenes Carneiro da Cunha- Instituto Federal de Educação Ciência e Tecnologia de

Alagoas /Brasil

\begin{abstract}
RESUMO: O número de pessoas com acesso à internet já chega a cerca de 116 milhões de usuários apenas no Brasil, e com isso, o número de adolescentes com problemas ocasionados pelo uso das redes sociais cresce aproximadamente na mesma intensidade. A ascensão da tecnologia tem auxiliado em diversas áreas como a da saúde, educação, segurança etc. Apesar dos benefícios deste recurso em tantas áreas, o seu uso desmedido tem gerado dependência, e afetado as relações sociais, causando consequências à saúde mental dos indivíduos, principalmente adolescentes e jovens. Profissionais afirmam que há relação entre o crescimento da depressão nos jovens e o aumento do tempo gasto em redes sociais; isso geralmente acontece devido à grande "vitrine virtual" apresentada nas redes, onde são exibidos corpos e personalidades "perfeitas". A presente pesquisa realizou uma ampla Revisão Sistemática de Literatura (RSL) com objetivo de examinar os impactos do uso das tecnologias digitais na saúde mental dos adolescentes. Foi seguido todo o protocolo da RSL como metodologia de pesquisa. E os resultados obtidos comprovaram que há relações entre a dependência tecnológica e a saúde psicológica de adolescentes e jovens. Também foi possível notar que as redes sociais virtuais podem acentuar problemas sociais e gerar grandes impactos na vida de qualquer pessoa, dentre eles: a ansiedade, depressão e dependência.
\end{abstract}

Palavras-chave: Adolescentes. Dependência. Depressão. Psicologia. Tecnologia.

\begin{abstract}
The number of people with internet access already reaches about 116 million users in Brazil alone, and with this, the number of teenagers with problems caused by the use of social networks grows at approximately the same intensity. The rise of technology has helped in many areas such as health, education, safety, etc. Despite the benefits of this resource in so many areas, its excessive use has generated dependence, and affected social relationships, causing consequences to the mental health of individuals, especially adolescents and young people. Professionals say there is a relationship between the growth of depression in young people and the increase in time spent on social networks; This is often due to the large "virtual showcase" displayed on the networks, where "perfect" bodies and personalities are displayed. This research conducted a comprehensive Systematic Literature Review (RSL) to examine the impacts of the use of digital technologies on adolescent mental health. The entire RSL protocol was followed as a research methodology. And the results obtained showed that there are relationships between technological dependence and psychological health of adolescents and young people. It was also noted that virtual social networks can accentuate social problems and generate major impacts on anyone's life, including anxiety, depression and dependence.
\end{abstract}

Keywords: Adolescents. Dependence. Depression. Psychology. Technology.

Educação, Psicologia e Interfaces, Volume 3, Número 3, p. 204-217, Setembro/Dezembro, 2019.

ISSN: 2594-5343. DOI: https://doi.org/10.37444/issn-2594-5343.v3i3.156 


\section{INTRODUÇÃO}

O século XXI trouxe a tecnologia como um auxiliar durante as relações sociais, sendo impensável viver sem ela nos dias atuais, especialmente para favorecer a comunicação através de ligações, e-mails, redes sociais, etc.

Silva e Silva (2017) afirmaram que o uso da internet todos os dias causa conflitos familiares, decorrentes da falta de diálogo, além disso, leva a relações superficiais, dificuldades de aprendizagem, transtornos de ansiedade e déficit de atenção. Já não se escreve cartas ou se utiliza o telefone convencional para conversar com o interlocutor, como antigamente. A grande maioria da comunicação tem sido através de redes sociais, que se tornou um poderoso instrumento na era da sociedade da informação.

Silva (2016) chamou a atenção para as redes sociais que se tornaram parte de nossas vidas. Na juventude, o uso da tecnologia pode tornar-se uma dependência, pois é onde os préjulgamentos são realizados, onde os relacionamentos são declarados e exibidos em "público", diante de pessoas muitas vezes desconhecidas. E sempre com o objetivo de alcançar o maior número de "amigos" com status manipulados por outros indivíduos, buscando incansavelmente obter o maior número de likes (curtidas) e comentários em suas postagens nas redes sociais. Não obstante, muitas vezes, chegam a excluir postagens por não alcançar um determinado público.

O uso problemático de mídias sociais revela um ambiente onde jovens chegam a agredir verbalmente pessoas com ideias e culturas diferentes de suas, que posteriormente podem causar danos psicológicos a outrem. O bullying virtual, as agressões verbais e as mensagens mal interpretadas podem influenciar em mudanças de hábito, discórdias e até desestruturação de famílias que são alguns dos fatores que aumentaram as taxas de quadros de depressão (LIN, 1973; SAMPASA-KANYINGA; HAMILTON, 2015; BLACHNIO et al. 2015; RADOVIV et al. 2017). A depressão apresenta sintomas que podem ser divididos em Sentimentos, Físicos, Pensamento e Comportamentais (DEL PORTO, 1999).

A relação entre a psicologia e a tecnologia digital foi descrita na teoria de Elias (1997), ressaltando que não há nenhuma possibilidade de o ser humano ser estudado, a não ser através de uma rede social da qual depende inteiramente. Atualmente o tema "redes sociais" tem sido relacionado a tecnologia digital, a internet, a dispositivos móveis. Diante de diversas visões, é notório que a tecnologia vem influenciando cada vez mais a vida dos jovens, assim como 
também é visível que atualmente há jovens se privando de uma vida social real em troca de uma virtual.

Este artigo visa apresentar uma Revisão Sistemática de Literatura (RSL) que analisou a relação entre casos de depressão e o frequente uso de aparelhos eletrônicos, internet e jogos etc. Os resultados desta análise foram norteados pela seguinte pergunta de pesquisa: Quais os impactos do uso excessivo das tecnologias digitais à saúde mental de adolescentes e jovens? Houve um planejamento voltado para expor os riscos do uso excessivo, maneiras de detecção de problemas à saúde mental e escalas de depressão.

\section{MATERIAL E MÉTODO}

Segundo Kitchenham et al (2009), uma RSL busca identificar, avaliar e interpretar os estudos que estejam disponíveis e que sejam importantes no contexto de determinadas questões de pesquisa. Esta seção apresenta as etapas de planejamento e de execução da RSL. O protocolo referente a esta pesquisa de revisão sistemática está disponível no link: https://drive.google.com/open?id=151IzFY84RIEGphnMHf4Y6djSEZs1oACa.

\subsection{Planejamento}

A presente pesquisa tem como objetivo responder à questão: "Quais os impactos do uso excessivo das tecnologias digitais à saúde mental de adolescentes e jovens?”. Para alcançar o objetivo deste estudo, foram formuladas outras três questões que serão abordadas no item a seguir. Os itens desta seção terão o objetivo de definir e apresentar as questões de pesquisa, bases de busca, critérios de seleção e strings utilizadas na pesquisa.

\subsubsection{Questões de pesquisa}

Kitchenham e Charters (2007) afirmaram que uma Revisão Sistemática de Literatura (RSL) é um estudo que utiliza uma metodologia bem definida para identificar, analisar e interpretar todas as evidências disponíveis a respeito de uma questão de pesquisa particular de maneira imparcial e Repetível. Com a definição anterior, é possível concluir que as questões de pesquisa são a base de uma RSL, pois é o que indica o que está sendo buscado, e norteia toda a pesquisa. Para esta RSL foram definidas três perguntas, são elas:

Q1. Quais os riscos do uso excessivo das redes sociais na vida dos adolescentes?

Educação, Psicologia e Interfaces, Volume 3, Número 3, p. 204-217, Setembro/Dezembro, 2019. ISSN: 2594-5343. DOI: https://doi.org/10.37444/issn-2594-5343.v3i3.156 
Q2. Como detectar problemas na saúde mental através de postagens e comportamentos na rede?

Q3. Quais as escalas para detecção de depressão?

\subsubsection{Bases de pesquisa}

Bases de pesquisa consistem nas fontes de busca onde foram coletadas as informações para responder as questões definidas. É importante destacar que a seleção das bases deve ser feita criteriosamente, buscando bases relacionadas ao campo de estudo da pesquisa.

A seleção das bases foi iniciada em uma fonte mais geral (Google Acadêmico), a partir daí, foram encontrados os materiais que levaram às outras 9 bases selecionadas, foram elas: Scielo, Bireme/BVS, PubMed, Science Direct, Repositório da Universidade Federal de Uberlândia, Repositório da Universidade do Porto, Repositório da Universidade de Lisboa, Repositório da Universidade de Brasília e Pepsic. Em sua maioria, os repositórios estão no campo da saúde/psicologia, e alguns dos repositórios são de Portugal, onde foram encontradas pesquisas sobre a temática do projeto.

\subsubsection{Critérios de seleção}

Os resultados da pesquisa irão depender dos estudos inseridos na RSL. De acordo com Sampaio e Mancini (2006) os critérios para incluir ou excluir um estudo devem ser especificados levando em consideração a questão de pesquisa, nisso incluem tempo, públicoalvo, intervenções, idioma, tipo de estudo, entre outros.

Com o intuito de alcançar bons resultados na busca foram criados os critérios de inclusão e de exclusão, onde no primeiro são informadas as características essenciais para que o material seja incluído, e no segundo, as características que fazem com que o material encontrado seja excluído da pesquisa. Para busca manual o ano de publicação deve ser de 2010-2017, o que altera o critério de inclusão 2. O Quadro 1 exibe os critérios de seleção utilizados nesta pesquisa.

Quadro 1. Critérios de seleção
\begin{tabular}{|c|l|}
\hline ID & \multicolumn{1}{|c|}{ Critérios de Inclusão (CI) } \\
\hline CI 1 & $\begin{array}{l}\text { Aponta problemas psicológicos e } \\
\text { tecnologia }\end{array}$ \\
\hline CI 2 & Publicado entre 2012 e 2017 \\
\hline CI 3 & Pesquisa com adolescentes/jovens. \\
\hline CI 4 & $\begin{array}{l}\text { Fala sobre a saúde mental de } \\
\text { adolescentes/jovens }\end{array}$ \\
\hline CI 5 & $\begin{array}{l}\text { Apresenta escalas de depressão/saúde } \\
\text { psicológica }\end{array}$ \\
\hline
\end{tabular}

\begin{tabular}{|c|l|}
\hline ID & \multicolumn{1}{|c|}{ Critérios de Exclusão (CE) } \\
\hline CE 1 & $\begin{array}{l}\text { Não está relacionado à influência da } \\
\text { tecnologia em problemas de saúde } \\
\text { psicológica }\end{array}$ \\
\hline CE 2 & $\begin{array}{l}\text { Não apresenta escalas (para a questão } \\
\text { 03) }\end{array}$ \\
\hline CE 3 & Resumo pouco compreensível \\
\hline
\end{tabular}




\begin{tabular}{|c|l|c|}
\hline ID & \multicolumn{3}{|c|}{ Critérios de Inclusão (CI) } \\
\hline CI 6 & $\begin{array}{l}\text { Foi realizada pesquisa com } \\
\text { adolescentes }\end{array}$ \\
\hline CI 7 & $\begin{array}{l}\text { Aborda o uso das redes sociais e } \\
\text { consequências de seu uso }\end{array}$ \\
\hline CI 8 & Aponta vícios tecnológicos & \\
\hline CI 9 & $\begin{array}{l}\text { Mostra relação a de } \\
\text { jovens/adolescentes com } \\
\text { tecnologias digitais }\end{array}$ & as \\
\hline
\end{tabular}

\begin{tabular}{|c|l|}
\hline ID & \multicolumn{1}{|c|}{ Critérios de Exclusão (CE) } \\
\hline CE 4 & $\begin{array}{l}\text { Fuga completa ou parcial do tema } \\
\text { proposto }\end{array}$ \\
\hline CE 5 & $\begin{array}{l}\text { Artigo não disponível para leitura } \\
\text { gratuita }\end{array}$ \\
\hline CE 6 & $\begin{array}{l}\text { Indisponível após etapa de seleção } \\
\text { por título }\end{array}$ \\
\hline
\end{tabular}

Fonte: Dados da pesquisa.

\subsubsection{Strings de busca}

As strings de busca são o conjunto de termos e palavras-chave que são utilizados na busca dos estudos, unidos por operadores lógicos - “OR” e "AND”, onde o primeiro significa que qualquer uma das palavras ou termos deve estar incluído na busca e o segundo, indica que o conjunto de palavras anterior deve ser incluso apenas se estiver acompanhado dos termos ou palavras seguintes. Moraes, Souza e Oliveira (2011) afirmaram que as strings podem ser adaptadas de acordo com o mecanismo de busca de cada Fonte, porém sempre de forma que não altere o seu sentido lógico. O Quadro 2 apresenta as strings, identificadas pelas questões de pesquisa.

\begin{tabular}{|c|c|}
\hline Quest. & String \\
\hline Q1 & $\begin{array}{l}\text { ("redes sociais" OR "redes sociais de internet" OR "redes sociais virtuais" OR "facebook" } \\
\text { OR "twitter" OR "instagram") AND ("adolescentes" OR "jovens") AND ("riscos" OR } \\
\text { "impactos" OR "vício" OR "dependência" OR "uso excessivo" OR "consequência") }\end{array}$ \\
\hline \multirow[t]{2}{*}{ Q2 } & $\begin{array}{l}\text { ("redes sociais" OR "redes sociais de internet" OR "redes sociais virtuais" OR "facebook" } \\
\text { OR "twitter" OR "instagram") AND ("adolescentes" OR "jovens") AND ("postagens" OR } \\
\text { "publicações" OR "divulgação" OR "compartilhamento" OR "comentário" OR "curtida" OR } \\
\text { "tweet" OR "retweet") AND ("comportamento" OR "emoções" OR "alteração de humor") } \\
\text { AND ("saúde mental" OR "distúrbio" OR "conflito emocional" OR "saúde psicológica" OR } \\
\text { "problemas mentais" OR "doença psicológica") AND ("detecção" OR "percepção" OR } \\
\text { "descoberta") }\end{array}$ \\
\hline & $\begin{array}{l}\text { ("rede social" OR "facebook" OR "instagram") AND ("adolescentes" OR "jovens") AND } \\
\text { ("postagens" OR "publicações" OR "compartilhamento") AND ("saúde mental" OR "saúde } \\
\text { psicológica" OR "doença psicológica") AND ("percepção" OR "descoberta") AND } \\
\text { (filetype:pdf) }\end{array}$ \\
\hline Q3 & $\begin{array}{l}\text { ("depressão" OR "saúde mental" OR "saúde psicológica" OR "distúrbio mental" OR } \\
\text { "ansiedade" OR "doença psicológica") AND ("detecção" OR "percepção" OR "descoberta") } \\
\text { AND ("escalas" OR "estratégias") }\end{array}$ \\
\hline
\end{tabular}

Fonte: Dados da pesquisa

$\mathrm{Na}$ Q2, a primeira string é a original, utilizada na maioria das bases de pesquisa, e a segunda foi a string adaptada para uma das bases de pesquisa (Google Acadêmico).

Educação, Psicologia e Interfaces, Volume 3, Número 3, p. 204-217, Setembro/Dezembro, 2019. ISSN: 2594-5343. DOI: https://doi.org/10.37444/issn-2594-5343.v3i3.156 


\subsection{Execução}

Durante a pesquisa, foram encontrados 9.953 trabalhos (artigos, dissertações, monografias, teses etc.) no total. Foi necessário dividir a fase de seleção em 2 etapas. $\mathrm{Na}$ primeira etapa, os materiais foram selecionados por título e palavras-chave, e alguns dos critérios de inclusão e exclusão puderam ser utilizados durante as filtragens das bases (ex.: CI2 e CE2), o que levou a 267 resultados.

Antes da segunda etapa, os resultados foram organizados em planilhas com as informações principais de cada um, e foram identificados por um ID. Além disso, foram identificados alguns resultados repetidos, alguns respondiam a mais de uma pergunta, e outros foram encontrados em diferentes bases. Ao reorganizar esta seleção, restaram 228 trabalhos.

$\mathrm{Na}$ segunda etapa os materiais foram separados pelos resumos, e foi possível aplicar os critérios de seleção. O resultado desta etapa foi de 27 trabalhos.

O Gráfico 1 apresenta a quantidade de trabalhos selecionados em cada base. A base de pesquisa que mais retornou resultados foi a BIREME/BVS com 10 trabalhos, seguida do Google Acadêmico com 7 resultados, 1 resultado esteve presente em duas bases, o Google Acadêmico com 07 trabalhos.

Gráfico 1. Quantidade de trabalhos por base de pesquisa.

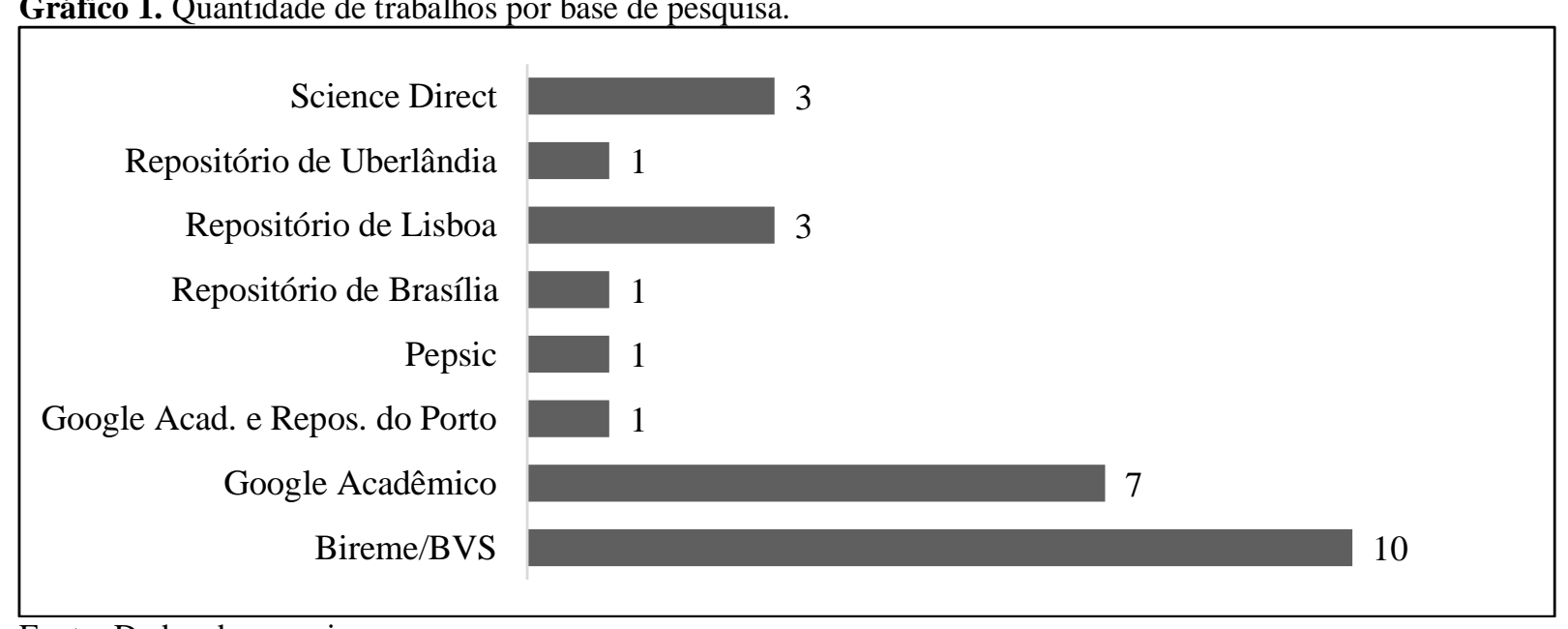

Fonte: Dados da pesquisa

O Gráfico 2, por sua vez, exibe a quantidade de trabalhos que apresentaram respostas para cada questão de pesquisa. Cabe ressaltar que alguns trabalhos também apontaram respostas para outras questões.

Gráfico 2. Contagem por questão de pesquisa 


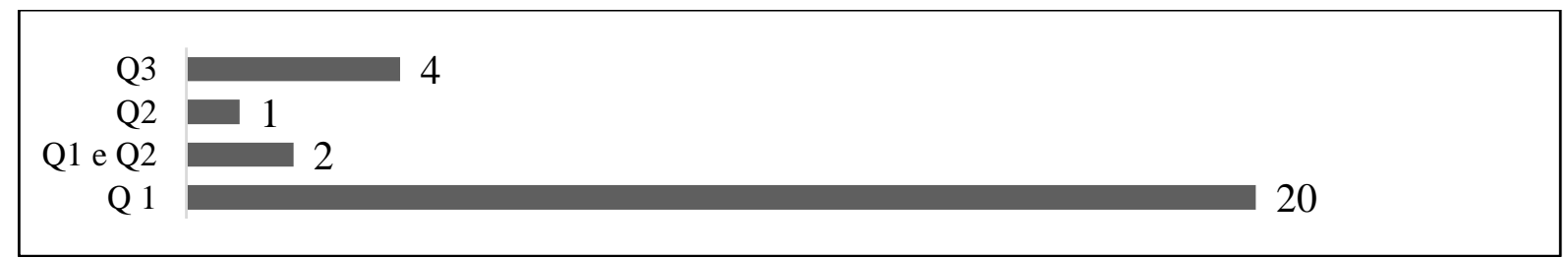

Fonte: Dados da pesquisa

Já no Gráfico 3, é apresentada a quantidade de trabalhos utilizados na RSL organizados por ano de publicação. Vale lembrar que o trabalho de 2010 foi resultado de busca manual, no Google Acadêmico, e não automática. É possível notar que a maioria dos trabalhos encontrados e utilizados são do ano de 2015.

\section{Gráfico 3. Quantidade de trabalhos selecionados por ano da publicação}

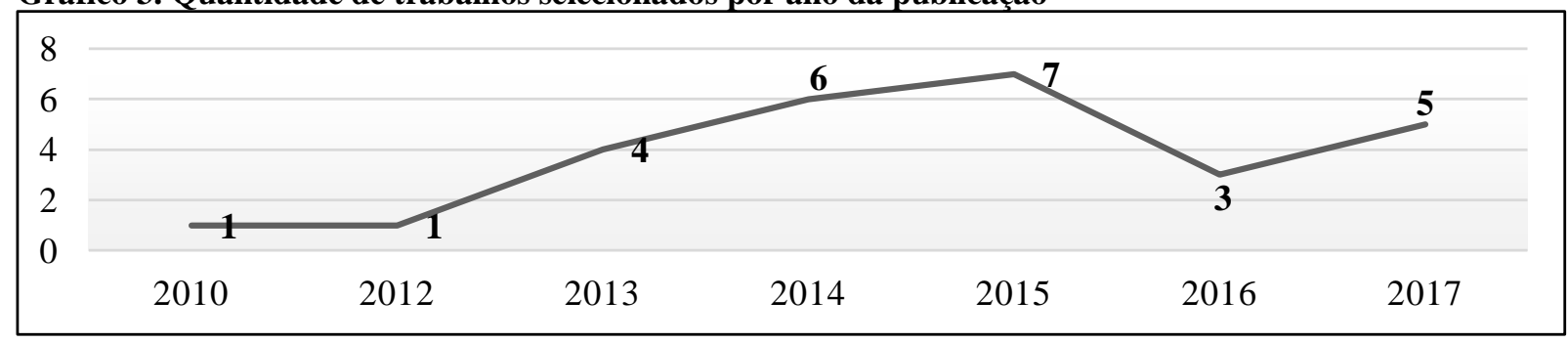

Fonte: Dados da pesquisa

\section{RESULTADOS E DISCUSSÃO}

A primeira questão (Q1) buscou identificar os riscos do uso excessivo das redes sociais na vida dos adolescentes. Após leitura dos trabalhos selecionados, foram obtidos os resultados apresentados na Figura 2, que retrata uma nuvem de palavras, onde quanto maior a fonte, maior o número de menções.

Figura 1. Nuvem de palavras - riscos do uso excessivo da tecnologia

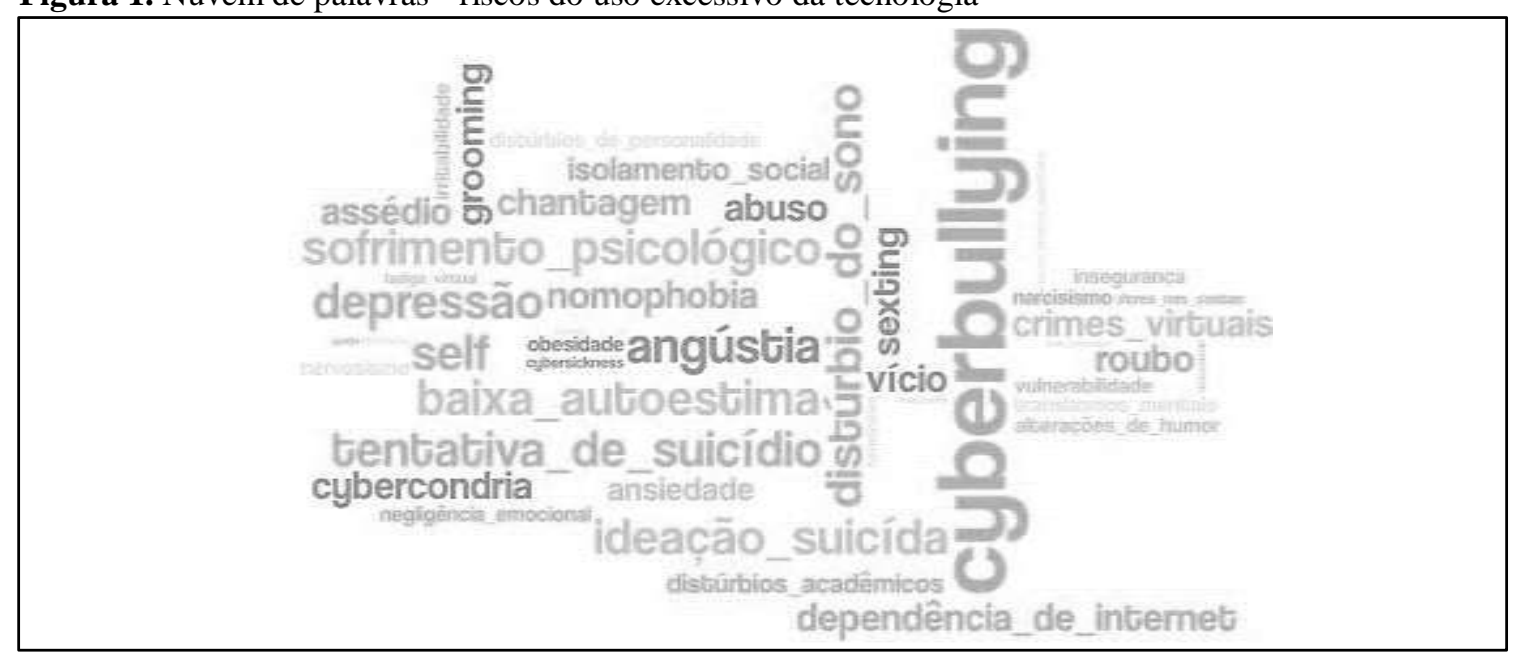

Fonte: Dados da pesquisa 
Ao analisar os riscos do uso excessivo das tecnologias digitais, com foco nas redes sociais virtuais, é possível destacar dois deles: cyberbullying (FRANCO e ARAGÓN, 2015; RICE et al. 2015; MUSSIO, 2017; CANHÃO, 2016; MAGAUD et al. 2013; SANTOS, 2015, RADOVIC et al. 2017; SAMPASA-KANYINGA e HAMILTON, 2015) e depressão (MUSSIO, 2017; CANHÃO, 2016; MARTINS, 2013; BLACHNIO et al. 2015; SAMPASAKANYINGA; HAMILTON, 2015; RADOVIC et al. 2017; PANTIC et al. 2012; CASH et al. 2013) - acompanhados também por características que direcionam a indícios de depressão. Também foram citados o suicídio, além de tentativas ou possíveis inclinações para o ato propriamente dito. Enquanto alguns autores citaram o cyberbullying e a depressão separadamente, Sampasa-Kanyinga e Hamilton (2015) afirmaram que há uma relação entre as vítimas de cyberbullying e os sintomas depressivos.

Para a segunda questão (Q2), a busca voltou-se para as maneiras de detectar problemas na saúde mental através de postagens e comportamentos. Após a leitura dos trabalhos que faziam alguma de menção a esta questão, foram obtidos os resultados exibidos no Quadro 4.

Quadro 3. Modos de detectar riscos/problemas para a saúde mental

\begin{tabular}{|l|c|}
\hline \multicolumn{1}{|c|}{ Deteç̧ão } & $\mathbf{N}^{\mathbf{o}}$ de citações \\
\hline $\begin{array}{l}\text { Passar mais tempo on-line, predisposição mais elevada para agredir e rendimento } \\
\text { acadêmico reduzido. }\end{array}$ & 1 \\
\hline Perda da capacidade de se concentrar. & 2 \\
\hline $\begin{array}{l}\text { Longas sessões noturnas online; mentir para familiares e amigos sobre o número de } \\
\text { horas que passa conectado. }\end{array}$ & 2 \\
\hline $\begin{array}{l}\text { Interações sociais negativas; postar conteúdo negativo; podem utilizar emoticons } \\
\text { em uma conversação escrita para denunciar o seu estado emocional real. }\end{array}$ & 2 \\
\hline Sintomas depressivos quando não tem o que postar. & 2 \\
\hline Exibir comportamentos de risco. & 2 \\
\hline Interage só via web; pode começar a desenvolver esquizofrenia por viver isolado. & 2 \\
\hline $\begin{array}{l}\text { Elevados problemas emocionais, usa a internet para regular o humor, ganhar apoio } \\
\text { social e liberar emoções. }\end{array}$ & 4 \\
\hline $\begin{array}{l}\text { Não consegue se desligar das redes sociais mesmo offline; o tempo dedicado à } \\
\text { ferramenta aumenta e o nível de controle que se tem sobre ela diminui. }\end{array}$ & 4 \\
\hline $\begin{array}{l}\text { Ansiedade por likes e comentários; fazem postagens sem pensar primeiro; } \\
\text { impaciência quando ficam sem acesso à Internet; apresenta abstinência e seus } \\
\text { efeitos; angústia e desconforto quando há impossibilidade de uso. }\end{array}$ & 4 \\
\hline $\begin{array}{l}\text { Uso compulsivo de internet; necessidade de estar conectado 24h por dia; são } \\
\text { obcecados e compelidos a checar constantemente o telefone; buscar rapidamente } \\
\text { conectar-se à Internet em qualquer lugar. }\end{array}$ & 4 \\
\hline Maior dificuldade em superar desafios; são agressivos e socialmente dominantes. & 4 \\
\hline $\begin{array}{l}\text { Ter poucos ou nenhum amigo real; ver a comunicação on-line mais segura e menos } \\
\text { ameaçadora; se esconder no anonimato do cyberespaço. }\end{array}$ & 5 \\
\hline Descrever métodos específicos de suicídio dentro do contexto de outros temas. & 9 \\
\hline Realizar postagens narcisistas. & 9 \\
\hline
\end{tabular}




\begin{tabular}{|l|c|}
\hline \multicolumn{1}{|c|}{ Detecção } & $\mathbf{N}^{\mathbf{0}}$ de citações \\
\hline $\begin{array}{l}\text { Oversharing (compartilhamento excessivo), postagens estressadas e posts de trigger } \\
\text { (acréscimo de contatos) de visualização. }\end{array}$ & 9 \\
\hline $\begin{array}{l}\text { Estão vulneráveis quanto a opinião dos outros; são dependentes de likes - sentem } \\
\text { necessidade de "curar" seus perfis. }\end{array}$ & 9 \\
\hline Reclamar de problemas situacionais. & 9 \\
\hline
\end{tabular}

Fonte: Dados da pesquisa

Entre o material analisado, nove apontaram que a maneira de detectar possíveis problemas à saúde mental está voltada às alterações de humor e comportamento, junto à grande necessidade de permanecer conectado mantendo seus perfis sempre atualizados e buscando cada vez mais seguidores (YOUNG; ABREU, 2011; RADOVIC et al. 2017; MUSSIO, 2017; SHERMAN et al. 2016; STEIN, 2013; CASH et al. 2013; FREITAS; SANTO, 2015; MEDRANO et al. 2017; ROSEN et al. 2012).

Dentre os trabalhos selecionados para responder a esta questão, dois deles, Silva e Silva (2017) e Faria (2015), apontaram oito sinais para auxiliar na identificação de dependência da Internet/Tecnologia Digital, foram eles: a preocupação excessiva com a internet, necessidade de aumentar o tempo conectado (on-line), presença de irritabilidade e/ou depressão, exibir esforços repetidos para diminuir o tempo de uso de internet, quando o uso de internet é reduzido apresenta labilidade emocional, permanecer mais conectado do que o programado, trabalhos e relações sociais em risco pelo uso excessivo, e mentir aos outros a respeito do tempo on-line.

A terceira questão (Q3) estava voltada para identificar as escalas para detectar a depressão. Após a análise dos trabalhos selecionados, foi possível notar que a escala mais utilizada é o Inventário de Depressão de Beck (BDI), esta escala foi citada nos trabalhos de Baptista et al (2010), Morais (2014) e Pantic et al (2012). No trabalho de Morais (2014) foram apresentadas escalas adaptadas para o público-alvo desta pesquisa, os adolescentes. As respostas obtidas estão dispostas no Gráfico 4.

Gráfico 4. Escalas para detecção de depressão

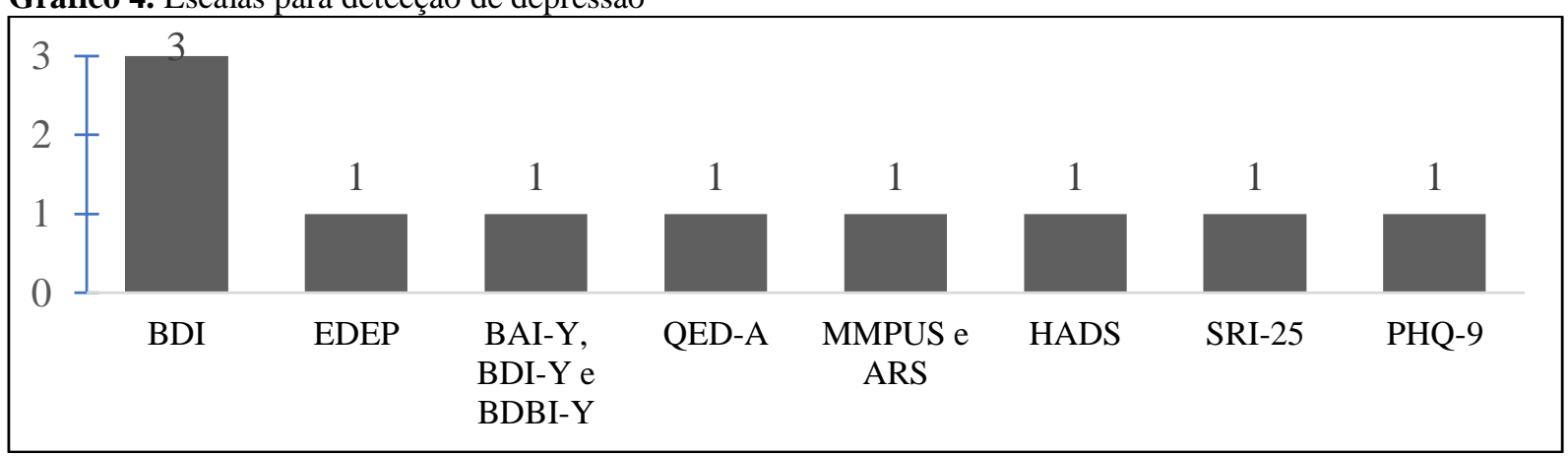

Fonte: Dados da pesquisa

Educação, Psicologia e Interfaces, Volume 3, Número 3, p. 204-217, Setembro/Dezembro, 2019. ISSN: 2594-5343. DOI: https://doi.org/10.37444/issn-2594-5343.v3i3.156 


\section{CONSIDERAÇÕES FINAIS}

Para responder à questão sobre os impactos do uso excessivo das tecnologias à saúde mental de adolescentes/jovens, é necessário explorar o campo da psicologia e compreender do que se trata a saúde mental, para em seguida efetuar a busca por sintomas e comportamentos apresentados nos jovens que utilizam as mídias sociais de maneira desenfreada, e só então fazer a comparação entre sintomas de doenças/transtornos mentais e os sintomas apresentados pelos indivíduos. Apesar de ser uma área de análise recente, a variedade de resultados - tanto para riscos como para os sintomas/comportamentos apresentados - é grande, como é possível observar nesta RSL. A leitura dos materiais selecionados nas buscas (automática e manual) possibilitou alcançar respostas para as questões definidas no início da revisão.

Para a Q1, os riscos apontados variaram entre taquicardia, alterações na respiração, tendinites e mudanças posturais (que são mais facilmente detectados), qualidade das relações familiares prejudicada, Cybersickness (náusea digital), vulnerabilidade afetiva, distúrbios alimentares, sedentarismo e obesidade, síndrome do toque fantasma (sensação de que o celular está tocando, sem que ele realmente esteja), narcisismo (preocupação completa com a própria imagem), distúrbios de personalidade, mudanças na auto-estima, distúrbios de concentração/acadêmicos, transtornos de ansiedade, fobia e isolamento social, dependências e vícios, crimes virtuais, grooming (assédio ou abuso sexual via mídias sociais de internet), distúrbios do sono, cyberbullying e selfie-cyberbullying, e por fim, depressão e suicídio.

Para a Q2, as maneiras para detectar possíveis problemas à saúde mental são o tempo que passam on-line - durante a pesquisa, foi visto que adolescentes envolvidos com cyberbullying passam mais tempo conectados do que os que não estão envolvidos -, problemas emocionais (agressividade, por exemplo) e sociais, rendimento acadêmico reduzido, postar e compartilhar conteúdo negativo ou comportamentos de risco, mentir sobre quanto tempo passa conectado, apresentam sintomas depressivos, utilizam as mídias sociais para melhorar o humor, ansiedade por likes (curtidas) e comentários ou para conectar-se à internet, mudanças de comportamento (inclusive mesmos comportamentos de dependentes químicos), uso compulsivo de internet, apresentam-se obcecados por checar constantemente o telefone, diminuí o contato direto com outros jovens e falta de amigos reais, vulneráveis quanto a opinião dos outros, e apresentam egoísmo exagerado em relação à tecnologia ( por exemplo). 
Para a Q3 foram obtidas as escalas EDEP, BDI, BAI-Y, BDI-Y, BDBI-Y, QED-A, MMPUS e ARS, HADS SRI-25, e PHQ-9. Estas escalas estão voltadas para detectar e/ou medir os níveis de depressão ou sintomas depressivos nos indivíduos.

Esta RSL apontou o cyberbullying e a depressão como principais riscos para o uso excessivo das tecnologias digitais; as alterações de humor e comportamento, e a grande necessidade de manterem-se conectados como principais maneiras de detectar problemas à saúde mental; e o BDI é a escala mais utilizadas para detectar depressão.

Essa análise é importante para chamar a atenção dos pais e educadores para a maneira como os jovens estão utilizando essas tecnologias, e para analisar se este uso está realmente sendo benéfico para a saúde mental deles.

Existem projetos para auxiliar no tratamento de nomofobia (dependência tecnológica), como o Instituto DELETE (um instituto de "Detox Digital", que orienta a população ao uso consciente das tecnologias digitais, e oferece suporte no tratamento) e o Grupo de Estudos sobre Adições Tecnológicas (GEAT - ajuda pais e educadores a estarem preparados para lidar com os desafios de educarem crianças e adolescentes em um mundo cada vez mais tecnológico).

Em trabalhos futuros, devem ser realizadas pesquisas de campo com adolescentes do ensino médio de escolas públicas e privadas, objetivando uma análise local escalável para confirmação e aplicação das informações obtidas nesta RSL.

\section{REFERÊNCIAS BIBLIOGRÁFICAS}

BAPTISTA, Makilim Nunes et al. Estudo psicométrico de escalas de depressão (EDEP e BDI) e o Inventário de Percepção de Suporte Familiar-IPSF. Revista Psicologia em Pesquisa, v. 4, n. 1, 2010.

BLACHNIO, A. et al. Internet use, Facebook intrusion, and depression: results of a crosssectional study. European Psychiatry, v. 30, n. 6, p. 681-684, 2015.

CANHÃO, Mariana Alves. Riscos e potencialidades do uso das redes sociais na adolescência. 2016. 27 f. Tese (Mestrado Integrado em Medicina) - Faculdade de Medicina, Universidade de Lisboa, Lisboa, 2016.

CASH, Scottye J. et al. Adolescent suicide statements on MySpace. Cyberpsychology, Behavior, and Social Networking, v. 16, n. 3, p. 166-174, 2013.

DEL PORTO, José Alberto. Conceito e diagnóstico. Revista Brasileira de Psiquiatria, v. 21, p. 06-11, 1999.

ELIAS, N. A sociedade dos indivíduos. Rio de Janeiro: Jorge Zahar, 1997. 
FARIA, Natyelle Gonçalves de. Fiz logout do mundo: dependência de redes sociais: patologia moderna ou nova forma de subjetividade?. 2015. $68 \mathrm{f}$. Trabalho de Conclusão de Curso (Graduação em Comunicação - Habilitação em Publicidade e Propaganda) - Escola de Comunicação, Universidade Federal do Rio de Janeiro, Rio de Janeiro, 2015.

FRANCO, Blanca E. Retana; ARAGÓN, Rozzana Sánchez. Acoso Cibernético: Validación en México del ORI-82. Acta de investigación psicológica, v. 5, n. 3, p. 2097-2111, 2015.

FREITAS, Ricardo Oliveira de; SANTO, Cíntia Sacramento do Espírito. A voz que vem da periferia: o jovem e suas narrativas percebidas na publicização de imagens e postagens nas redes sociais. Pontos de Interrogação-Revista de Crítica Cultural, v. 4, n. 1, p. 11-38, 2015.

HORMES, Julia M.; KEARNS, Brianna; TIMKO, C. Alix. Craving F acebook? Behavioral addiction to online social networking and its association with emotion regulation deficits. Addiction, v. 109, n. 12, p. 2079-2088, 2014.

KITCHENHAM, B. et al. Systematic literature reviews in software engineering - A systematic literature review. ELSEVIER, v.51, p. 7-15, 2009.

KITCHENHAM, B.; CHARTERS, S. Guidelines for performing systematic literature reviews in software engineering. Technical Report EBSE 2007-001, Keele University and Durham University Joint Report (2007).

LIN, TY. L'étude epidémologique de troubles mentaux. In: Seminaire de L'OMS surl'organisation dês services da Santè Mentale. 1973, Addis-Abeba.

MAGAUD, Emilie; NYMAN, Karissa; ADDINGTON, Jean. Cyberbullying in those at clinical high risk for psychosis. Early intervention in psychiatry, v. 7, n. 4, p. 427-430, 2013.

MARTINS, Daniela Alves. Adolescentes internautas, família, e depressão: Estudo da relação entre a utilização da internet e das redes Sociais, o ambiente familiar e a sintomatologia depressiva. 86 p. Dissertação (Mestrado em Psicologia) - Universidade de Lisboa, Faculdade de Psicologia, Lisboa, 2013.

MEDRANO, José Luis Jasso; ROSALES, Fuensanta López; LOVING, Rolando Díaz. Conducta adictiva a las redes sociales y su relación con el uso problemático del móvil. Acta de Investigación Psicológica, v. 7, n. 3, p. 2832-2838, 2017.

MORAES, Taciano Messias; SOUZA, Adriana Silveira de; OLIVEIRA, Juliano Lopes de. Revisão sistemática sobre a comunicação dentro do processo de desenvolvimento de software. Workshop Anual do MPS, 7, 2011, Campinas, SP: SOFTEX, 2011, 124-134.

MORAIS, Résia Silva de. Inventários Beck para crianças e adolescentes: revisão sistemática da literatura. 113 f. Dissertação (Mestrado em Ciências Humanas) Universidade Federal de Uberlândia, Uberlândia, 2014.

MUSSIO, Rogéria Albertinase Pincelli. A geração Z e suas respostas comportamental e emotiva nas redes sociais virtuais. Dissertação de Mestrado (Pós-graduação em 
Desenvolvimento Humano e Tecnologias - IBRC) - Universidade Estadual Paulista - Instituto de Biociências de Rio Claro, Rio Claro, SP, 2017.

PANTIC, Igor et al. Association between online social networking and depression in high school students: behavioral physiology viewpoint. Psychiatria Danubina, v. 24, n. 1., p. 9093, 2012.

RADOVIC, Ana; et al. "Depressed Adolescents' Positive and Negative Use of Social Media." Journal of adolescence 55 (2017): 5-15. PMC.

RICE, Eric et al. Cyberbullying perpetration and victimization among middle-school students. American Journal of Public Health, v. 105, n. 3, p. e66-e72, 2015.

ROSEN, LD; et al. Is Facebook creating "iDisorders"? The link between clinical symptoms of psychiatric disorders and technology use, attitudes and anxiety. Comput Human Behav. 2012; 29(3): 1243-54.

SAMPAIO, RF; MANCINI, MC. Estudos de revisão sistemática: um guia para síntese criteriosa da evidência científica. Revista brasileira de fisioterapia, v. 11, n. 1, p. 83-89, 2006.

SAMPASA-KANYINGA, H.; HAMILTON, H. A. Social networking sites and mental health problems in adolescents: The mediating role of cyberbullying victimization. European psychiatry, v. 30, n. 8, p. 1021-1027, 2015.

SANTOS, Mariana Ferreira Teixeira. Cyberbullying na adolescência: perfil psicológico de agressores, vítimas e observadores. 95 p. Dissertação (Mestrado Integrado em Psicologia) Universidade de Lisboa, Lisboa, 2015.

SHERMAN, Lauren E. et al. The power of the like in adolescence: Effects of peer influence on neural and behavioral responses to social media. Psychological science, v. 27, n. 7, p. 1027-1035, 2016.

SILVA, Ana Paula Areias da. As implicações do uso da rede social Facebook para a felicidade dos adolescentes. Dissertação (Mestrado em Gestão Comercial) - Faculdade de Economia, Universidade do Porto, Portugal.

SILVA, Thayse de Oliveira; SILVA, Lebiam Tamar Gomes. Os impactos sociais, cognitivos e afetivos sobre a geração de adolescentes conectados às tecnologias digitais. Rev.

psicopedag., São Paulo, v. 34, n. 103, p. 87-97, 2017. Disponível em:

$<$ http://pepsic.bvsalud.org/scielo.php?script=sci_arttext\&pid=S0103-

$\underline{84862017000100009 \& \operatorname{lng}=p t \& n r m=i s s o>}$. Acessos em jan 2018.

STEIN, Joel. Millenials: the me me me generation. Time, [S. 1.], 9 maio 2013. Disponível em: https://time.com/247/millennials-the-me-me-me-generation/. Acesso em: 07 jul. 2018.

YOUNG, Kimberly S.; ABREU, Cristiano Nabuco de. Dependência de Internet: Manual e Guia de Avaliação e Tratamento. Porto Alegre, Editora Artmed, 2011. 


\section{Credenciais das autoras}

SOUZA, Karlla. Instituto Federal de Educação Ciência e Tecnologia de Alagoas /Brasil. Email: karlladanielly94@hotmail.com

CUNHA, Mônica Ximenes Carneiro da. Mestrado em Engenharia Elétrica pela Universidade Federal de Campina Grande e doutorado em Administração pela Universidade Federal de Pernambuco. É professora do Instituto Federal de Educação Ciência e Tecnologia de Alagoas. E-mail: mxccunha@gmail.com

Endereço para correspondência: Karlla Souza E-mail: karlladanielly94@ @otmail.com

Como citar este artigo (Formato ABNT): SOUZA, Karlla; CUNHA, Mônica Ximenes Carneiro da. Impactos do uso das redes sociais virtuais na saúde mental dos adolescentes: uma revisão sistemática da literatura. Educação, Psicologia e Interfaces, v. 3, n.3, p. 204217, 2019. DOI: https://doi.org/10.37444/issn-2594-5343.v3i3.156

Recebido: 07/08/2019.

Aceito: 20/11/2019. 